\title{
LS-Based Pilot Contamination Pre-coding for Large-scale MIMO System
}

\author{
Jiang Jing, Wang Ni and Xu Shuang \\ Xi'an University of Posts \& Telecommunications, Xi'an, China \\ jiangjing18@gmail.com,lemontree626808@163.com, smile5236@126.com
}

\begin{abstract}
To reduce pilot contamination, a downlink precoding algorithm was put forward for Large-scale MIMO system. It set the Least Square $(L S)$ criterion to the objective function, and then solved the precoding matrices with the Lagrange multiplier method. In the solving, it utilized not only the estimated channel information but also the pilot contamination information, which was acquired from some blank resource elements, so could more precisely reduce pilot contamination. Based on simulation results, it was shown that the proposed algorithm can achieve better performance than popular Large-scale MIMO precoding algorithms, e.g. single-cell Zero-Forcing precoding and multi-cell MMSE precoding. It could break through the limit of pilot contamination and acquire approximately linear performance improvement with the number of antennas increasing.
\end{abstract}

Key words: Large-scale MIMO, Precoding, Pilot Contamination, Least Square

\section{Introduction}

Large-scale MIMO (also known as Massive MIMO) is an advanced and attractive technology for $5 \mathrm{G}$ wireless communication. The base station boosts the number of antennas from several tens to several hundreds. With the gain of large-scale antenna array, large-scale MIMO significantly improves the cell throughput, suppresses interference and reduces the base station transmit power, and is called "green communications" [1]. From the point of view of information theory, the channel capacity tends to the infinity when transmitting and receiving antennas increase infinitely. However, the performance of large-scale MIMO is limited by Pilot Contamination [2,3]. To avoid a large amount of the overhead MIMO channel information feedback, Large-scale MIMO mainly works in TDD mode and utilizes the uplink channel measurement to compute the downlink precoding matrix based on the assumption of the channel reciprocity [4]. The channel information is increased greatly with the number of antenna increasing, uplink pilots may be reused in adjacent cells. Pilot contamination is caused by the uplink channel estimation which is polluted by reused or non-orthogonal pilots of users from other cells. The downlink large-scale MIMO precoding matrixes are computed on the polluted uplink channel estimation; it ultimately degrades the performance of the system [5].

Therefore, pilot contamination has become the key issue in the research of large-scale MIMO. There are two main solutions in the current study. The first solution is enhancements on pilot orthogonal design. [6-8] put forward that interfering cells should transmit pilots to stagger in time/frequency/code domains and combine the pilot transmission schemes with power control in order to reduce pilot contamination. However, for multi-cell system, the measured channel information of every user is increasing with the number of antennas 
increasing, and several users are multiplexed on the same physical resources, it is rather difficult to ensure transmitting pilots orthogonally [9]. Proposed a channel estimation method on Bayesian criterion to obtain second-order statistics channel information, e.g., the average arrival angle and the angle spread of the arrival angle and then coordinately schedule uplink users in adjacent cells which have the smaller pilots contamination. It eventually reduces the pilot contamination and improves the channel capacity. The second solution is pilot contamination precoding scheme. Assuming the system model with a single cell, $[10,11]$ proposed ZF (zero forcing), MRC (maximum ratio combining) and MMSE (Minimum Mean Square Error) and other linear precoding algorithms and compared their performance. Simulation results show that the performance of large-scale MIMO system is far superior to the single antenna system. Contrasts to the above analysis of the pilot contamination, those algorithms haven't considered this issue. Document [12] described the large-scale MIMO capacity considering pilot contamination and proposed multi-cell MMSE (Minimum Mean Square Error) precoding algorithm. The objective function of MMSE was divided into two parts: the variance of the cell channel estimation and the second other-cell interference order statistics. It used the expectations of the sum of these two parts as the optimization objective function to obtain a multi-cell precoding matrix [13]. Introduced a precoded pilot contamination (PCP) concept, the base station linearly combined the slow fading coefficient of terminal to each pilot multiplexing cell into a slow fading coefficient matrix, and the product of the matrix and the conventional single -cell linear precoding matrix is the pilot pollution precoding matrix.

[12] Directly used the estimated channel instead of the real channel, the real channel contains estimated information, pilot interference information and noise. The channel information of the pilot contamination cell wasn't exploited, so the improvement of the performance is limited. On the other hand, in large-scale MIMO system, the downlink beam is accurately aligned, so the inter-user interference could be negligible in the downlink transmission [2]. This paper focuses on the impact of pilot contamination to reduce the complexity of the algorithm. The downlink precoding algorithm based on least squares (LS) criteria of detected signal and the transmitted signal was put forward, and the precoding matrix was calculated with Lagrange multiplier method. The innovation of this paper is to acquire the pilot contamination channel information from a spot of blank resource elements, then solve the precoding matrices with the information, so could more precisely reduce pilot contamination. Based on simulations results, it was shown that the proposed algorithm can reduce the impact of pilot pollution on the performance of downlink precoding and achieve better performance than traditional precoding algorithms, e.g., single-cell Zero-Forcing precoding and multi-cell MMSE precoding.

\section{System Model}

Aassumed that Large-scale MIMO system works on TDD mode. The channel reciprocity can be exploited in this system, namely using formula: $\boldsymbol{H}=\overrightarrow{\boldsymbol{H}}^{\mathrm{H}}$, downlink channel $\overrightarrow{\boldsymbol{H}}$ can be acquired from the uplink channel training $\boldsymbol{H}$ in the coherence time.

There are $I$ cells in Large-scale MIMO system in which $L$ cells use non-orthogonal and even the same pilot sequences. One base station of each cell is equipped with $M$ antennas and the user equipment of per cell configures single antenna. To simplify the description, in the uplink training resource, OFDM symbol ${ }^{t}$ and subcarrier $\omega$ will all be assigned to user

$k$ of per cell. The uplink training processing is shown in Figure 1, the received signal ${ }^{y_{j}}$ of cell ${ }^{j}$ for OFDM symbol ${ }^{t}$ and subcarrier $\omega$ is denoted as following: 


$$
\boldsymbol{y}_{j}=\boldsymbol{H}_{j j} \boldsymbol{x}_{j}+\sum_{l=1}^{L} \boldsymbol{H}_{l, \neq j} \boldsymbol{x}_{j}+\sum_{i=i \notin l,}^{I} \boldsymbol{H}_{j i} \boldsymbol{x}_{i}+\boldsymbol{n}_{j}
$$

Where $\boldsymbol{H}_{i j}$ is an $M \times 1$ channel matrix from user $k$ of cell $j$ to the base station of cell $j$ and $\boldsymbol{H}_{j j}=\sqrt{\beta_{i j}} h_{j j} . \quad \beta_{j j}$ Is a real coefficient of the large-scale fading and shadow fading and $\boldsymbol{h}_{j j}$ is an $M \times 1$ fast fading matrix whose elements are independent and identically distributed (i.i.d.) with $C N\left(0, \boldsymbol{I}_{M}\right), \boldsymbol{H}_{j l}$ is the channel matrix from the $k$ th user of cell $l$ to the base station of cell $j$ and $\boldsymbol{H}_{j i}$ is the channel matrix from the $k$ th user of cell $i$ to the base station of cell $j . x_{j}$ Are uplink pilots used by user $k$ of cell $l$. $\boldsymbol{x}_{i}$ Are uplink pilots used by user $k$ of cell $i$ and orthogonal with $x_{j}$ and $x_{i} x_{j}^{\mathrm{H}}=\delta_{i j}$. The sequence length of uplink pilots is $\tau, n_{j}$ is the additive white Gaussian noise of cell $j$ and $\boldsymbol{n}_{j j} \in C N(0,1)$.

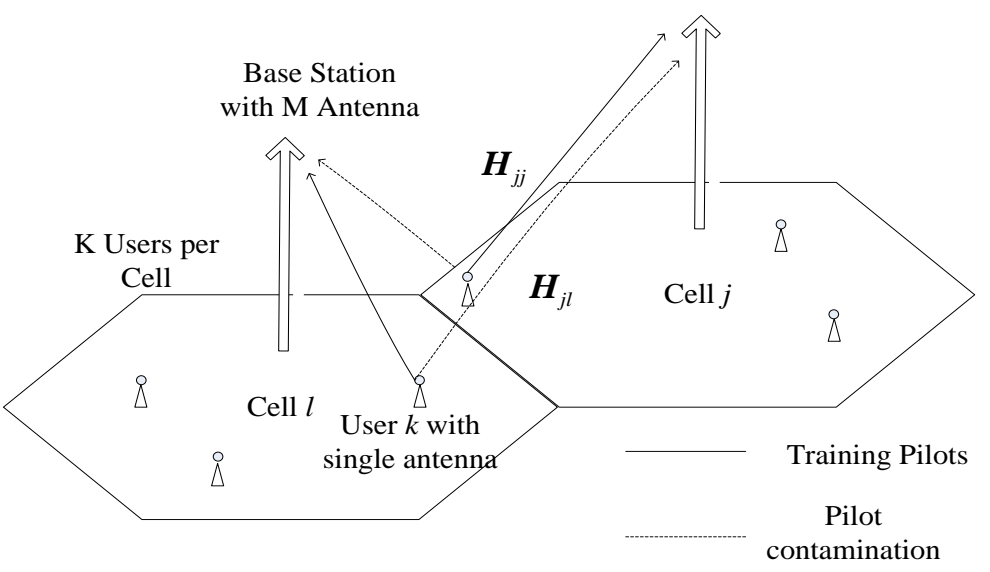

Figure 1. Uplink System Model of Massive MIMO

From (1), the LS (Least Square) estimate value $\hat{\boldsymbol{H}}_{i j}$ of the channel $\boldsymbol{H}_{j j}$ is obtained from the following formula:

$$
\hat{\boldsymbol{H}}_{j j}=\boldsymbol{y}_{j} \cdot \boldsymbol{x}_{j}^{H} / \sqrt{p_{r} \tau}=\boldsymbol{H}_{j j}+\sum_{l=1, l \neq j}^{L} \boldsymbol{H}_{j l}+\boldsymbol{n}_{j}^{\prime}
$$

Where $\boldsymbol{n}_{j}^{\prime}=\frac{\boldsymbol{n}_{j} x_{j}^{\mathrm{H}}}{\sqrt{p_{r} \tau}}, \quad \boldsymbol{n}_{j}^{\prime} \in C N\left(0, \frac{\boldsymbol{I}_{M}}{p_{r} \tau}\right)$. So the estimated channel $\hat{\boldsymbol{H}}_{j j}$ contains real channel $\boldsymbol{H}_{j j}$, pilot contamination channel $\sum_{l=1, l \neq j}^{L} \boldsymbol{H}_{j l}$ and the noise. If estimated channel $\hat{\boldsymbol{H}}_{j j}$ is directly utilized to downlink MIMO precoding, it would reduce the accuracy of MIMO processing and finally degrade the performance gains of large-scale MIMO.

\section{LS-based Precoding Algorithm to Reduce Pilot Contamination}

The basic idea of the proposed algorithm is that the pilot contamination is considered in large-scale MIMO precoding processing. Through optimizing the precoding matrix, the 
performance degradation caused by the pilot contamination would be avoided. Assumed that the downlink received signal $\boldsymbol{r}_{j}$ of user $k$ cell $j$ can be written as:

$$
\boldsymbol{r}_{j}=\sum_{l=1}^{L} \boldsymbol{H}_{j l} \boldsymbol{W}_{l} \boldsymbol{s}_{l}+\sum_{i=1, i \neq l}^{I} \boldsymbol{H}_{j i} \boldsymbol{W}_{i} \boldsymbol{s}_{i}+\boldsymbol{z}_{j}
$$

Where $s_{l}$ is the transmitting signal? $\boldsymbol{H}_{j l}$ is the channel matrix considering the transmitting power and large-scale fading factor, and $\boldsymbol{H}_{j l}=\sqrt{p_{f} \beta_{j l}} \boldsymbol{h}_{j l}$. The channel estimation $\hat{\boldsymbol{H}}_{j l}=\sqrt{p_{f} \beta_{j l}} \hat{\boldsymbol{h}}_{j l}$, so the channel estimation error is $\tilde{\boldsymbol{H}}_{j l}=\hat{\boldsymbol{H}}_{j l}-\boldsymbol{H}_{j l} \cdot z_{j}$ is the white Gaussian noise and the power is equal to $1 . W_{j}$ is the downlink precoding matrix.

In order to reduce the impact of pilot contamination on the downlink precoding performance, the linear precoding algorithm based on LS criterion is proposed in this paper. Minimize the sum of square error between the normalized detecting signal and the sending signal. Because there is a single antenna at receiver, it can be written as:

$$
\min _{\boldsymbol{w}_{l}, \alpha_{l}}\left\|\tilde{\boldsymbol{s}}_{l}-\boldsymbol{s}_{l}\right\|^{2}=\min _{\boldsymbol{w}_{l}, \alpha_{l}}\left\|\alpha_{l} \boldsymbol{r}_{j}-\boldsymbol{s}_{l}\right\|^{2}
$$

Where $\alpha_{l}$ is a scaling factor. Moreover, because the transmitted beam of each cell in large-scale MIMO system aims at the target user more accurately, the interference between users is negligible [1][2]. In order to reduce the computational complexity, interference term $\sum_{i=1, i \neq l}^{I} \boldsymbol{H}_{j i} \boldsymbol{W}_{i} \boldsymbol{s}_{i}$ is zero in this paper and then formula (4) can be written as:

$$
\min _{\boldsymbol{W}_{l}, \alpha_{l}}\left\|\alpha_{l} \boldsymbol{r}_{j}-\boldsymbol{s}_{l}\right\|^{2}=\min _{\boldsymbol{W}_{l}, \alpha_{l}}\left\|\alpha_{l}\left(\boldsymbol{H}_{l l} \boldsymbol{W}_{l} \boldsymbol{s}_{l}+\boldsymbol{z}_{l}\right)-\boldsymbol{s}_{l}\right\|^{2}
$$

In the practical solution, the real channel $\boldsymbol{H}_{j l}$ can't be obtained. According to $\boldsymbol{H}_{j l}=\hat{\boldsymbol{H}}_{j l}-\tilde{\boldsymbol{H}}_{j l}$, we use the difference of estimated channel value $\hat{\boldsymbol{H}}_{j l}$ and estimation error instead of the real channel in the solving process. And the channel estimation error can be acquired from a small amount of blank resource elements in the uplink pilot measurement. Users didn't send the pilot of the cell in the resource elements; only measure the information of other-cell pilot interference and noise. According to formula (2), for cell $l$, the optimization problems of precoding matrix based on LS criterion can be expressed as:

$$
\begin{gathered}
\min _{\boldsymbol{W}_{l}, \alpha_{l}}\left\|\alpha_{l}\left(\hat{\boldsymbol{H}}{ }_{l l} \boldsymbol{W}_{l} \boldsymbol{s}_{l}-\sum_{j=1, j \neq l}^{L} \boldsymbol{H}_{l j} \boldsymbol{W}_{l} \boldsymbol{s}_{l}-\boldsymbol{n}_{l}^{\prime} \boldsymbol{W}_{l} \boldsymbol{s}_{l}+\boldsymbol{z}_{l}\right)-\boldsymbol{s}_{l}\right\|^{2} \\
\text { s.t. } \operatorname{tr}\left\{\boldsymbol{W}_{l}{ }_{l}^{H} \boldsymbol{W}_{l}\right\}=1 .
\end{gathered}
$$

The optimization objective function is $J\left(\boldsymbol{W}_{l}, \alpha_{l}\right)$, then formula (6) further can be written as: 


$$
\begin{aligned}
\boldsymbol{J}\left(\boldsymbol{W}_{l}, \alpha_{l}\right)= & \left\|\alpha_{l}\left(\hat{\boldsymbol{H}}{ }_{l l} \boldsymbol{W}_{l} \boldsymbol{s}_{l}-\sum_{j=1, j \neq l}^{L} \boldsymbol{H}_{l j} \boldsymbol{W}_{l} \boldsymbol{s}_{l}-\boldsymbol{n}_{l}^{\prime} \boldsymbol{W}_{l} \boldsymbol{s}_{l}+z_{l}\right)-\boldsymbol{s}_{l}\right\|^{2} \\
= & \left\|\left(\alpha_{l} \hat{\boldsymbol{H}}_{l l} \boldsymbol{W}_{l}-\alpha_{l} \sum_{j=1, j \neq l}^{L} \boldsymbol{H}_{l j} \boldsymbol{W}_{l}-\alpha_{l} \boldsymbol{n}_{l}^{\prime} \boldsymbol{W}_{l}-\boldsymbol{I}_{K}\right) \boldsymbol{s}_{l}\right\|+\alpha_{l}^{2} \\
= & \left(\alpha_{l} \boldsymbol{W}_{l}{ }_{l}\left(\hat{\boldsymbol{H}}_{l l}-\sum_{j=1, j \neq l}^{L} \boldsymbol{H}_{l j}-\boldsymbol{n}_{l}^{\prime}\right)^{H}-\boldsymbol{I}_{K}\right)\left(\alpha_{l}\left(\hat{\boldsymbol{H}}_{\| l}-\sum_{j=1, j \neq l}^{L} \boldsymbol{H}_{l j}-\boldsymbol{n}_{l}^{\prime}\right) \boldsymbol{W}_{l}-\boldsymbol{I}_{K}\right)+\alpha_{l}^{2} \\
= & \alpha_{l}{ }_{l} \boldsymbol{W}_{l}{ }_{l}\left\|\hat{\boldsymbol{H}}_{l l}-\sum_{j=1, j \neq l}^{L} \boldsymbol{H}_{l j}-\boldsymbol{n}_{l}^{\prime}\right\|^{2} \boldsymbol{W}_{l}-\alpha_{l} \boldsymbol{W}_{l}{ }_{l}\left(\hat{\boldsymbol{H}}_{l l}-\sum_{j=1, j \neq l}^{L} \boldsymbol{H}_{l j}-\boldsymbol{n}_{l}^{\prime}\right)^{H}- \\
& \alpha_{l}\left(\hat{\boldsymbol{H}}_{\| l}{ }^{-} \sum_{j=1, j \neq l}^{L} \boldsymbol{H}_{l j}-\boldsymbol{n}_{l}^{\prime}\right) \boldsymbol{W}_{l}+\alpha_{l}^{2}+1
\end{aligned}
$$

Using the Lagrange method for solving the optimal solution, the associated Lagrangian is:

$$
L\left(\boldsymbol{W}_{l}, \alpha_{l}, \lambda\right)=J\left(\boldsymbol{W}_{l}, \alpha_{l}\right)+\lambda\left(\operatorname{tr}\left\{\boldsymbol{W}_{l}{ }_{l}^{H} \boldsymbol{W}_{l}\right\}-1\right)
$$

Where $\lambda$ is a Lagrange multiplier, assuming

$$
\begin{gathered}
\boldsymbol{Q}=\left(\hat{\boldsymbol{H}}_{l l}-\sum_{j=1, j \neq l}^{L} \boldsymbol{H}_{l j}-\boldsymbol{n}_{l}^{\prime}\right)^{H}\left(\hat{\boldsymbol{H}}_{l l}-\sum_{j=1, j \neq l}^{L} \boldsymbol{H}_{l j}-\boldsymbol{n}_{l}^{\prime}\right)+\frac{\lambda}{\alpha_{l}^{2}} \boldsymbol{I}_{M}, \text { the following formula can be obtained: } \\
L\left(\boldsymbol{W}_{l}, \alpha_{l}, \lambda\right)=\left\|\alpha_{l} \boldsymbol{Q}^{\frac{1}{2}} \boldsymbol{W}_{l}-\boldsymbol{Q}^{-\frac{1}{2}}\left(\hat{\boldsymbol{H}}_{l l}-\sum_{j=1, j \neq l}^{L} \boldsymbol{H}_{l j}-\boldsymbol{n}_{l}^{\prime}\right)^{H}\right\|^{2}- \\
\operatorname{tr}\left\{\left(\hat{\boldsymbol{H}}_{l l}-\sum_{j=1, j \neq l}^{L} \boldsymbol{H}_{l j}-\boldsymbol{n}_{l}^{\prime}\right) \boldsymbol{Q}^{-1}\left(\hat{\boldsymbol{H}}_{l l}-\sum_{j=1, j \neq l}^{L} \boldsymbol{H}_{l j}-\boldsymbol{n}_{l}^{\prime}\right)\right\}+\alpha_{l}^{2}+1-\lambda
\end{gathered}
$$

Thus, the last three of the formula (9) are independent of $\boldsymbol{W}_{1}$, when $\alpha_{l} \boldsymbol{Q}^{\frac{1}{2}} \boldsymbol{W}_{l}=\boldsymbol{Q}^{-\frac{1}{2}}\left(\hat{\boldsymbol{H}}_{l l}-\sum_{j=1, j \neq l}^{L} \boldsymbol{H}_{l j}-\boldsymbol{n}_{l}^{\prime}\right)^{H}$, the results of the above formula are minimum. The optimal solution can be written as:

$$
\boldsymbol{W}_{l}^{o p t}=\frac{1}{\alpha_{l}} \boldsymbol{Q}^{-1}\left(\hat{\boldsymbol{H}}_{l l}-\sum_{j=1, j \neq l}^{L} \boldsymbol{H}_{l j}-\boldsymbol{n}_{l}^{\prime}\right)^{H}
$$

Where $\alpha_{l}^{o p t}$ satisfied the formula: $\operatorname{tr}\left\{\left(\boldsymbol{W}_{l}^{o p t}\right)^{H} \boldsymbol{W}_{l}^{o p t}\right\}=1$.

\section{The Analysis of Simulation Results}

In this section, we will use simulation to evaluate the performance of the proposed pilot contamination precoding algorithm. At the same time, we compared the proposed algorithm with two popular precoding algorithms: single cell ZF (zero forcing) precoding [2] and multi-cell MMSE precoding. The precoding matrix of single cell ZF is obtained 
by $\hat{\boldsymbol{H}}_{u}^{H}\left(\hat{\boldsymbol{H}^{H}} \hat{\boldsymbol{H}}_{u}\right)^{-1}$, and the precoding matrix of MMSE is shown in literature [12]. Other simulation parameters are shown in Table 1.

Table 1. Simulation Parameters

\begin{tabular}{ll}
\hline Simulation parameters & Values \\
\hline Carrier frequency /Bandwidth & $2.4 \mathrm{GHz} / 10 \mathrm{MHz}$ \\
\hline Subcarrier spacing & $15 \mathrm{KHz}$ \\
Antenna spacing & $0.5 \lambda$ \\
Multi-cell & Three cells \\
Transmission power & Uplink: $p_{\tau}=10 \mathrm{~dB}$, Downlink: $p_{f}=20 \mathrm{~dB}$ \\
& Scenario 1, Scenario 2 \\
Large- scale fading coefficient & BS: 100 antennas UE: 1 antenna \\
Antenna configuration & Independent and identically distributed Rayleigh fading channel \\
Channel model & 20000 bit \\
The number of bits & Adaptive modulation and coding \\
Modulation and coding scheme & LS channel estimation \\
Channel estimation &
\end{tabular}

For the simulation of multi-cell interference and pilot contamination, it's assumed that there are three cells. The pilot of cell 1 and cell 2 are common, simultaneously orthogonal with the pilot of cell 3. In order to more fully reflect the performance of the proposed algorithm, two scenarios were simulated. Scenario 1: the large-scale fading coefficient of cell 1 is $\beta_{1}=0.8$, the large- scale fading coefficient of cell 2 is $\beta_{2}=0.1$, and the large-scale fading coefficient of cell 3 is $\beta_{3}=0.1$; Scenario 2 : the large-scale fading coefficient of cell 1 is $\beta_{1}=0.2$, the large- scale fading coefficient of cell 2 is $\beta_{2}=0.2$, and the large-scale fading coefficient of cell 3 is $\beta_{3}=0.6$, cell 2 is one third of cell 3 . So in scenario 1 , multi-cell interference and pilot contamination is weak, but rather heavy in scenario 2 .

The simulation results are shown in Figures 2 and 3. It can be seen, as the number of antennas increases, the performance of single-cell ZF algorithm rises slowly because of pilot contamination and other cell interference. In Figure 3, due to the serious pilot contamination, increasing of the number of antennas cannot improve performance. It's shown that the performance of large-scale MIMO systems is limited by the pilot contamination, which is consistent with the analysis of literatures $[2,12]$. In Figures 2 and 3, it's shown that multi-cell MMSE precoding and the proposed algorithm is better than single-cell ZF precoding for considering the pilot contamination mitigation. In the scheme 2 of serious pilot contamination, precoding performance improvement is more obvious compared with the single cell ZF. With the increasing of the number of antennas, the spectral efficiency of these two algorithms increases. It's proved that these two algorithms can significantly reduce the degradation on the performance of the pilot contamination.

Because the proposed algorithm does not consider the interference from other cells, the performance advantages are less obvious when the number of antennas is 20; but with the increasing of the number of antennas, impact of inter-cell interference can be ignored when channel between users tend to be more orthogonality. The algorithm of this paper can be more precise to eliminate the inter-cell interference, so performance improved approximate linearly. 


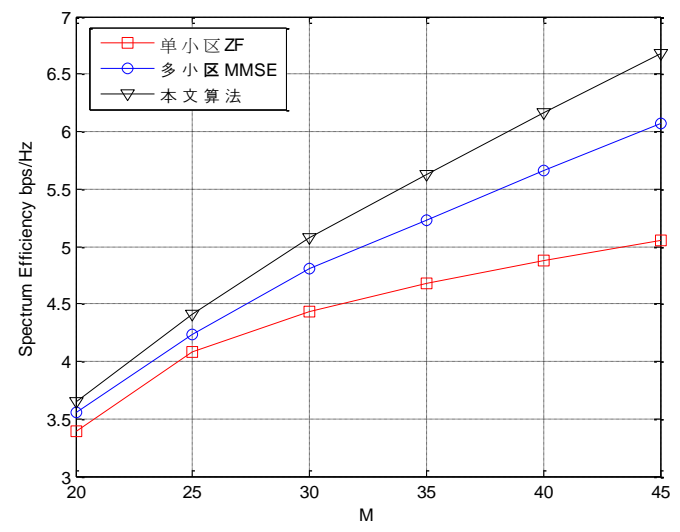

Figure 2. Simulation Results of Pilot Pollution Precoding (Scenario 1)

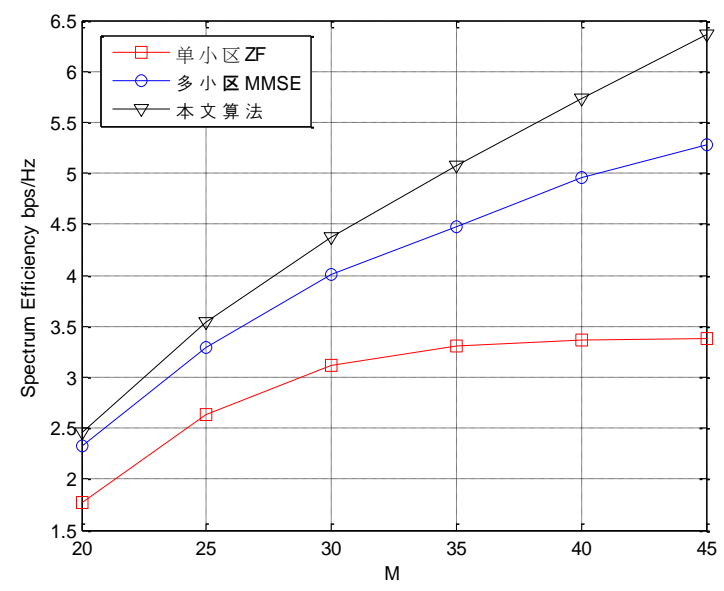

Figure 3. Simulation Results of Pilot Pollution Precoding (Scenario 2)

\section{Conclusion}

We took full advantage of the channel estimation information of local cell and the pilot contamination channel in order to reduce the impact of pilot contamination on the performance of downlink precoding. Simulation results proved that the proposed algorithm can reduce the performance limits of pilot contamination, its performance improved approximate linearly with the increasing of SNR. In future studies, the situation which the number of user antennas is larger than 1 can be considered. At the same time, we can combine the idea of pilot contamination reducing precoding with SVD, SLNR and other existing popular linear precoding algorithm and extend to single-user multi-stream or multi-user multi-stream application scenarios.

\section{Acknowledgements}

This paper is sponsored by the National Natural Science Foundation of China (61102047) and the Natural Science Foundation of Shanxi Province (2014JM8320). 


\section{References}

[1] T. L. Marzetta, "Non cooperative cellular wireless with unlimited numbers of base station antennas". IEEE Trans. Wireless Commun, vol. 9, no. 11, (2010).

[2] F. Rusek, D. Persson, B. K. Lau, E. G. Larsson, T. L. Marzetta, O. Edfors and F. Tufvesson, "Scaling up MIMO: Opportunities and challenges with very large arrays.IEEE Signal Process. Mag, vol. 30, no. 1, (2012).

[3] J. Hoydis and S. T. Brink, "Massive MIMO in the UL/DL of Cellular Networks: How Many Antennas Do We Need?", IEEE Journal On Selected Areas In Communications, vol. 31, no. 2, (2013).

[4] T. L. Marzetta, "How much training is required for multiuser MIMO?". IEEE Asilomar Conference on Signals, Systems and Computers (ACSSC'06), (2006), pp. 359-363.

[5] J. Jose, A. Ashikhmin and T. L. Marzettaet, "Pilot contamination problem in multi-cell TDD systems", IEEE International Symposium on Information Theory (ISIT’09), (2009), pp. 2184-2188.

[6] W. Hairong, W. Yuhui, H. Yongming and Y. lvxi, "Pilot Contamination Reduction in Very Large MIMO Multi-Cell TDD Systems", Journal of signal processing, vol. 29, no. 2, (2013).

[7] K. Appaiah, A. Ashikhmin and T. L. Marzetta, "Pilot Contamination Reduction in Multi-User TDD Systems", IEEE International Conference on Communications (ICC), (2010), pp. 1-5.

[8] B. Gopalakrishnan and N. Jindal, "An analysis of pilot contamination on multi-user MIMO cellular systems with many antennas", IEEE International Workshop on Signal Processing Advances in Wireless Communications (SPAWC), (2011), pp. 381-385.

[9] H. Yin, D. Gesbert, M. Filippou and Y. Liu, "A Coordinated Approach to Channel Estimation in Large-Scale Multiple-Antenna Systems", IEEE Journal on Selected Areas in Communications, vol. 31, no. 2, (2013).

[10] X. Gao, F. Tufvesson and E. F. Rusek, "Measured propagation characteristics for very-large MIMO at 2.6 GHz", European Conference on Antennas and Propagation (EUCAP), (2012), pp. 433-437.

[11] H. Q. Ngo, E. G. Larsson and T. L. Marzetta, "Energy and spectral efficiency of very large multiuser MIMO systems", IEEE Trans. Commun., vol. 61, no. 4, (2012).

[12] J. Jose, A. Ashikhmin, T. L. Marzettaet, et al., "Pilot Contamination and Precoding in Multi-Cell TDD Systems", IEEE Trans. Wireless Commun., vol. 10, no. 8, (2011).

[13] A. Ashikhmin and T. L. Marzettaet, "Pilot Contamination Precoding in Multi-Cell Large Scale Antenna Systems", IEEE International Symposium on Information Theory .Proceedings, (2012).

[14] O. N. Airabadi, E. Tsakalaki and H. Huang, "Beamforming via Large and Dense Antenna Arrays Above a Clutter”, IEEE Journal on Selected Areas in Communications, vol. 31, no. 2, (2013).

[15] F. Fernandes, A. Ashikhmin and T. L. Marzetta, "Inter-Cell Interference in Non cooperative TDD Large Scale Antenna Systems", IEEE Journal on Selected Areas in Communications, vol. 31, no. 2, (2013).

\section{Authors}

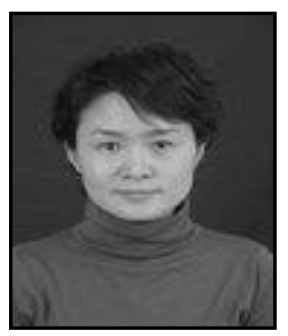

Jiang Jing, she received M. Sc. from the XiDian University in 2005 and a Ph.D. in Information and Communication Engineering from North Western Polytechnic University, China in 2009. She had been a researcher and a project manager from 2006 to 2012 at ZTE Corporation in China, and currently as an associate professor of School of Communication and Information Engineering in Xi'an University of Posts \& Telecommunications, China. Her research interests include wireless communication theory and MIMO system design. She has been a Member of the IEEE, 3GPP.

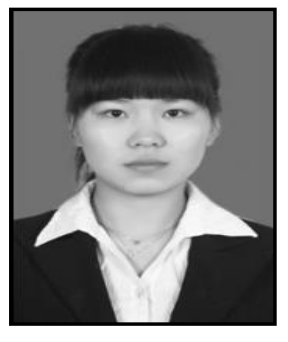

Wang Ni, she was born in Shanxi Province, China, in 1988. She received the B.E. degree from School of Communication and Information Engineering in Xi'an University of Posts \& Telecommunications, in 2012. She is currently studying as a M.E. in School of Communication and Information Engineering in Xi'an University of Posts \& Telecommunications, China. Her research interests include Massive MIMO System, Heterogeneous Network System. 


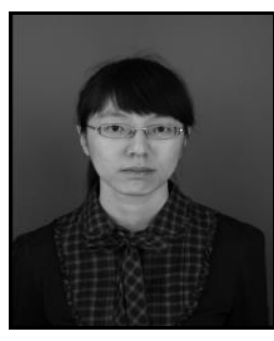

Xu Shuang, she was born in Shanxi Province, China, in 1989. She received the B.E. degree from Tibet University for Nationalities, Xianyang in 2012. She is currently studying as a M.E. in School of Communication and Information Engineering in Xi'an University of Posts \& Telecommunications, China. Her research interests include channel information feedback for Massive MIMO. 
International Journal of Future Generation Communication and Networking Vol.7, No.4 (2014) 